\title{
Discinese escapular em atletas de esportes acima da cabeça: revisão integrativa
}

\author{
Scapular Dyskinesis in above head sports athletes: integrative review \\ Discinesis escapular en atletas deportivos por encima de la cabeza: revisión integrativa
}

Recebido: 29/11/2021 | Revisado: 03/12/2021 | Aceito: 10/12/2021 | Publicado: 18/12/2021

\author{
Mirelly Miranda Nogueira \\ ORCID: https://orcid.org/0000-0003-2537-5696 \\ Faculdade Independente do Nordeste, Brasil \\ E-mail: mirellymn@outlook.com \\ Virgílio Santana Júnior \\ ORCID: https://orcid.org/0000-0003-1069-2584 \\ Faculdade Independente do Nordeste, Brasil \\ E-mail: virgiliofisio@hotmail.com
}

\begin{abstract}
Resumo
$\mathrm{O}$ atleta possui grandes demandas de treino, que se intensificam durante as temporadas esportivas. Treinos excessivos tendem a sobrecarregar as estruturas responsáveis pela formação do movimento, quanto maior a sobrecarga de uma estrutura, maior o risco de lesão e dor, amplificando o risco de afastamento do esporte. Atletas com modalidades esportivas que envolvem arremesso repetitivos podem gerar alteração na movimentação da escapula em relação ao ombro, sendo uma das alterações comuns a discinese escapular, que pode ser descrita como o mau posicionamento da escapula durante os movimentos do ombro ou em repouso. Este estudo tem como objetivo analisar, através da literatura científica, o índice da discinese escapular em atletas arremessadores. Trata-se de uma revisão integrativa da literatura, realizada através do levantamento de artigos científicos que abordassem a relação entre discinese escapular em corredores através das bases de dados: Pubmed, Scielo, Lilacs e Medline, publicados entre os anos de 2016 e 2021. A estratégia de busca totalizou 90 artigos potencialmente relevantes, sendo realizada a revisão do título e do resumo, apenas 10 artigos restaram, sendo esses retidos e lidos na íntegra. Por fim, 3 artigos foram considerados relevantes e retidos para análise. Os resultados demonstraram que a discinese escapular pode aumentar o risco para lesões de ombro em atletas. A discinese escapular comumente está presente em esportes específicos para membros superiores, sendo que idade, volume de treinamentos e cargas podem aumentar as chances de lesões no membro superior quando comparados a atletas sem alterações na movimentação escapular.
\end{abstract}

Palavras-chave: Discinese escapular; Lesão no esporte; Atleta de arremesso.

\begin{abstract}
The athlete has great training demands, which intensify during the sporting seasons. Excessive training tends to overload the structures responsible for the formation of movement, the greater the overload of a structure, the greater the risk of injury and pain, amplifying the risk of withdrawing from the sport. Athletes with sports that involve repetitive throwing can cause changes in the movement of the scapula in relation to the shoulder, one of the common changes being scapular dyskinesis, which can be described as malposition of the scapula during shoulder movements or at rest. This study aims to analyze, through the scientific literature, the scapular dyskinesis index in throwing athletes. This is an integrative literature review, carried out through a survey of scientific articles that addressed the relationship between scapular dyskinesis in runners through the following databases: Pubmed, Scielo, Lilacs and Medline, published between 2016 and 2021. A search strategy totaled 90 potentially relevant articles, with the title and abstract being reviewed, only 10 articles remained, which were retained and read in full. Finally, 3 articles were considered relevant and retained for analysis. The results demonstrated that scapular dyskinesis can increase the risk for shoulder injuries in athletes. Scapular dyskinesis is commonly present in specific sports for upper limbs, and age, training volume and loads can increase the chances of upper limb injuries when compared to athletes without changes in scapular movement.
\end{abstract}

Keywords: Scapular dyskinesis; Injury in sport; Throwing athlete.

\section{Resumen}

El deportista tiene grandes exigencias de entrenamiento, que se intensifican durante las temporadas deportivas. El entrenamiento excesivo tiende a sobrecargar las estructuras responsables de la formación del movimiento, cuanto mayor es la sobrecarga de una estructura, mayor es el riesgo de lesión y dolor, amplificando el riesgo de retirarse del deporte. Los deportistas con deportes que implican lanzamientos repetidos pueden provocar cambios en el movimiento de la escápula en relación con el hombro, siendo uno de los cambios comunes la discinesia escapular, que puede describirse como una mala posición de la escápula durante los movimientos del hombro o en reposo. Este estudio tiene como objetivo analizar, a través de la literatura científica, el índice de discinesia escapular en deportistas 
de lanzamiento. Se trata de una revisión integradora de la literatura, realizada a través de una encuesta de artículos científicos que abordaron la relación entre discinesia escapular en corredores a través de las siguientes bases de datos: Pubmed, Scielo, Lilacs y Medline, publicados entre 2016 y 2021. Una estrategia de búsqueda totalizó 90 potencialmente relevantes artículos, con el título y el resumen en revisión, solo quedaron 10 artículos, los cuales fueron retenidos y leídos en su totalidad. Finalmente, 3 artículos se consideraron relevantes y se conservaron para su análisis. Los resultados demostraron que la discinesia escapular puede aumentar el riesgo de lesiones en el hombro en los atletas. La discinesia escapular suele estar presente en deportes específicos para las extremidades superiores, y la edad, el volumen de entrenamiento y las cargas pueden aumentar las posibilidades de lesiones en las extremidades superiores en comparación con los atletas sin cambios en el movimiento escapular.

Palabras clave: Discinesia escapular; Lesión en el deporte; Atleta lanzador.

\section{Introdução}

$\mathrm{O}$ ato de arremessar requer geração de força máxima em um curto período de tempo, que devem ser otimizados para atingir potência e precisão, exigindo altos números de repetições para atingir consistência de desempenho, deixando esses atletas expostos ao risco de lesões por esforço repetitivo, além disso predispõem lesões por alto estresse em tendões, músculos, ligamentos e articulações. Lesões no ombro, foram destacadas como uma área que justifica esforços preventivos em uma ampla variedade de esportes de arremesso, onde o ombro é exposto a grandes demandas devido a movimentos repetidos acima da cabeça em alta velocidade. Problemas e dores no ombro representam uma carga significativa na área esportiva, cerca de $19 \%$ a $36 \%$ dos jogadores de handebol relatam queixa de dor no ombro no início da temporada e $28 \%$ durante a temporada (Moller, 2017; Andersson, 2016; Meron, 2017).

Redução na rotação interna e excesso de rotação externa da glenoumeral, alteração de força em manguito rotador, variação da amplitude de movimento e presença de discinese escapular foram relatados como fatores de risco para problemas no ombro (Moller, 2017; Andersson, 2016). Na busca por uma melhor performance o atleta exigi muito do corpo, podendo acarretar em lesões, essa por sua vez é a causa mais comum para abandono da prática esportiva mediante a incapacidade de se exercitar devido à dor ou desconforto. A dor possui impacto negativo sobre o esportista, afetando o desempenho, atividade do treino e vida diária (Moller, 2017; Mulyad, 2018).

A oscilação do braço durante a marcha desempenhar auxilio na recuperação do equilíbrio após uma perturbação e diminuição do gasto energético. O papel da escápula é fornecer uma base estável e maximizar os graus de liberdade necessários para execução dos movimentos dos membros superiores (MMSS). A discinesia escapular é caracterizada por mau posicionamento escapular durante o repouso e movimento, sinal de instabilidade e é resultado de fraqueza dos músculos escapulo torácicos (Hejrati, 2016; Nodehi, 2020; Romkes, 2017; McQuade, 2016). Frente ao exposto, esta pesquisa apresenta como objetivo analisar, através da literatura científica, o índice da discinese escapular em atletas arremessadores.

\section{Materiais e Métodos}

Trata-se de uma revisão integrativa da literatura, segundo Ercole (2014), esse tipo de estudo tem como finalidade sintetizar resultados obtidos em pesquisas sobre determinada problemática, de modo sistemático, ordenada e abrangente. Percorrendo seis etapas: identificação do tema através da seleção da hipótese; critérios de inclusão e exclusão para busca na literatura; definição das informações a serem extraídas dos estudos selecionados; avaliação dos estudos incluídos; interpretação dos resultados; e apresentação da síntese do conhecimento.

A pesquisa foi realizada através do levantamento de artigos científicos que abordassem a relação entre discinese escapular em corredores através das bases de dados: Pubmed, Scielo, Lilacs e Medline. Foram utilizados descritores (DeCS) na língua inglesa, sendo utilizadas as palavras: "Athletic Injuries", "Scapula" e "Scapular Dyskinesis". Os descritores foram utilizados em combinação "Scapular Dyskinesis" e "Athletic Injuries", "Scapula"e "Ahletic Injurues”, através dos operadores boleanos "and". 
Os critérios de inclusão foram jogadores de esportes acima da cabeça, estar disponível em texto completo, escrito em inglês e ser publicado entre os anos de 2016 e 2021. Os critérios de exclusão do estudo: experimentação em animais, não escritos em inglês, não disponíveis no texto completo, livros, dissertações, teses, monografias, anais de eventos e os artigos de revisão tendo em vista que o presente estudo também é uma pesquisa de revisão.

\section{Resultados}

A estratégia de busca totalizou 90 artigos potencialmente relevantes no Pubmed $(n=53)$, Scielo $(n=2)$, Lilacs $(n=4)$ e Medline $(\mathrm{n}=31)$. Após a revisão do título e do resumo, apenas 10 artigos foram retidos e lidos na íntegra. Após a triagem dos 10 artigos, 7 foram excluídos da análise. Por fim, 3 artigos foram considerados relevantes e retidos para análise. Esta estratégia de pesquisa é descrita em Figura 1.

Figura 1.

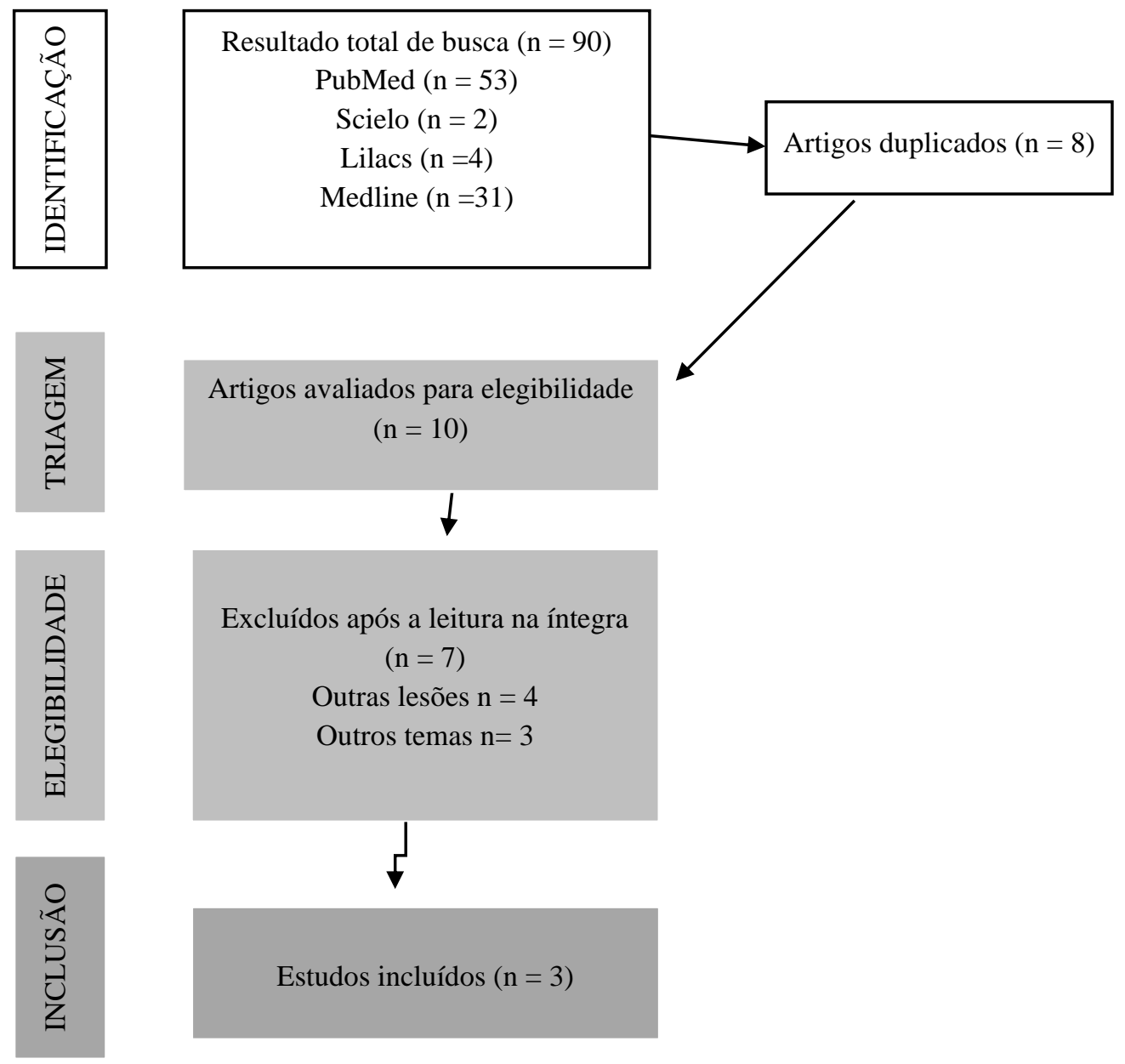

Fonte: Dados da pesquisa.

As características dos artigos incluídos nesta revisão estão resumidas em Quadro 1, contendo as seguintes informações: título do artigo, autor, metodologia e resultados. A amostra combinada foi de 1.818 participantes (961 jogadores de tênis, 679 jogadores de handebol e 178 de outras modalidades). Houve um estudo de coorte, uma revisão sistemática e um estudo transversal. A amostra de participação dos estudos foi de jogadores de tênis de diferentes idades, gêneros e níveis de 
habilidade e praticantes das seguintes modalidades esportivas: voleibol, natação, handebol, basquete, judô e jogadores de handebol juvenil.

Quadro 1.

\begin{tabular}{|c|c|c|c|c|}
\hline $\begin{array}{c}\text { Título do } \\
\text { artigo }\end{array}$ & Autor e ano & Metodologia & Objetivo & Conclusão \\
\hline $\begin{array}{l}\text { Handball load } \\
\text { and shoulder } \\
\text { injury rate: a } \\
\text { 31-week } \\
\text { cohort study } \\
\text { of } 679 \text { elite } \\
\text { youth } \\
\text { handball } \\
\text { players }\end{array}$ & $\begin{array}{l}\text { Moller et al, } \\
2016\end{array}$ & $\begin{array}{l}\text { Estudo de coorte, composto por } 679 \\
\text { jogadores (14-18 anos) forneceram } \\
\text { relatórios semanais sobre lesões no } \\
\text { ombro e carga de handebol (horas de } \\
\text { treinamento e competição) durante } 31 \\
\text { semanas usando o sistema de SMS, } \\
\text { telefone e exame médico. A carga de } \\
\text { handebol em uma determinada semana } \\
\text { foi categorizada em <20\% de aumento } \\
\text { ou diminuição, aumento entre } 20 \% \text { e } \\
60 \% \text { e aumento de> } 60 \% \text { em relação à } \\
\text { quantidade média semanal de carga de } \\
\text { handebol nas } 4 \text { semanas anteriores. }\end{array}$ & $\begin{array}{l}\text { Investigar a relação do } \\
\text { aumento de carga semanal } \\
\text { em jogadores de handebol } \\
\text { adolescentes de elite e } \\
\text { taxas de lesão no ombro e } \\
\text { se essa associação é } \\
\text { influenciada pelo controle } \\
\text { escapular, força isométrica } \\
\text { do ombro e Amplitude de } \\
\text { movimento da glenoumeral }\end{array}$ & $\begin{array}{l}\text { Um grande aumento na carga } \\
\text { semanal de handebol níveis } \\
\text { acima de } 20 \% \text {, aumenta a taxa } \\
\text { de lesões no ombro em } \\
\text { jogadores de handebol juvenil } \\
\text { de elite, principalmente se } \\
\text { esses tiverem presença de } \\
\text { redução da força rotacional } \\
\text { externa ou discinesia } \\
\text { escapular. }\end{array}$ \\
\hline $\begin{array}{l}\text { Scapular } \\
\text { dyskinesis } \\
\text { was not } \\
\text { associated } \\
\text { with pain and } \\
\text { function in } \\
\text { male } \\
\text { 4dolescente } \\
\text { athletes }\end{array}$ & $\begin{array}{l}\text { Oliveira et } \\
\text { al, } 2018\end{array}$ & $\begin{array}{l}\text { Estudo transversal, composto por } 178 \\
\text { adolescentes do sexo masculino } \\
(14,58 \pm 2,16 \text { anos). Aplicação do } \\
\text { questionário Quick Disability Arm } \\
\text { Shoulder Hand, avaliação da massa } \\
\text { corporal, estatura, rotação interna do } \\
\text { ombro e Closed Kinetic Chain Upper } \\
\text { Extremity Stability. Para avaliação da } \\
\text { discinese escapular, utilizou-se o } \\
\text { método visual dinâmico. A comparação } \\
\text { entre os grupos com e sem discinese foi } \\
\text { feita pelo teste te Mann-Whitney, sendo } \\
\text { aceito significância estatística quando } \\
\text { p<0,05. }\end{array}$ & $\begin{array}{l}\text { Estimar a prevalência da } \\
\text { discinese escapular em } \\
\text { atletas amadores jovens. } \\
\text { Além de, avaliar a } \\
\text { associação entre discinese } \\
\text { escapular e dor no ombro e } \\
\text { investigar se essa condição } \\
\text { influencia na função do } \\
\text { ombro dos atletas. }\end{array}$ & $\begin{array}{l}\text { A discinese escapular não está } \\
\text { associada à dor e não altera } \\
\text { medidas de função no ombro } \\
\text { em adolescentes atletas. } \\
\text { Modalidade, idade e volume } \\
\text { de treinamento parecem } \\
\text { induzir mudanças na } \\
\text { movimentação escapular. }\end{array}$ \\
\hline $\begin{array}{l}\text { Risk factors } \\
\text { for Upper } \\
\text { Limb Injury } \\
\text { in Tennis } \\
\text { Players: A } \\
\text { systematic } \\
\text { review }\end{array}$ & $\begin{array}{c}\text { Kekelekis et } \\
\text { al, } 2020\end{array}$ & $\begin{array}{l}\text { Revisão sistemática foi conduzida de } \\
\text { acordo com os itens de relatório } \\
\text { preferidos para revisões sistemáticas e } \\
\text { meta-análises (PRISMA), usando uma } \\
\text { questão de pesquisa desenvolvida pelo } \\
\text { problema do paciente, (ou população) } \\
\text { intervenção, comparação ou controle, e } \\
\text { metodologia Outcome (PICO). }\end{array}$ & $\begin{array}{l}\text { Identificar e avaliar as } \\
\text { evidências que relacione os } \\
\text { fatores de risco para lesão } \\
\text { de membro superior em } \\
\text { tenistas. }\end{array}$ & $\begin{array}{l}\text { A maioria das lesões do tênis } \\
\text { está associada ao uso } \\
\text { excessivo e ao curso crônico } \\
\text { do tempo, entretanto, as lesões } \\
\text { do tênis não surgem de uma } \\
\text { combinação linear de fatores } \\
\text { isolados e preditivos. São } \\
\text { fatores de risco para lesão de } \\
\text { membro superiores: discinesia } \\
\text { escapular, fadiga muscular, } \\
\text { propriedades } \\
\text { escapulotorácicas, cinética ou } \\
\text { cinemática do ombro, nível de } \\
\text { habilidade e técnica. }\end{array}$ \\
\hline
\end{tabular}

Fonte: Dados da pesquisa.

\section{Discussão}

Alterações da cinemática escapular são definidas como discinesia escapular, caracterizadas por um mau posicionamento escapular durante o repouso e alteração na movimentação, além disso pessoas sintomáticas costumam apresentar dor inespecífica no ombro. Estudos corroboram sobre a hiperativação do trapézio superior com inibição do trapézio médio/inferior e serrátil anterior (Huang, 2018; Nowotny, 2018). Dentre os fatores causais encontramos, problemas ósseos, problemas neurológicos, problemas de tecidos moles e inflexibilidade muscular, sendo as últimas as razões mais comuns para movimento anormal de ombro. Ressaltando que a discinese pode ser a causa de patologias degenerativas do ombro, sendo fator de risco para síndrome do impacto, instabilidade da glenoumeral, fratura clavicular, distúrbio do manguito rotador, lesão labial superior e patologia da articulação acromioclavicular (Giuseppe, 2020; Borms, 2020). 
Atletas com discinese escapular e diminuição da força rotacional externa até os $30^{\circ}$ possuem modificação no efeito de adaptação ao aumento de carga do handebol, quando excedem 20-60\% ficam mais propensos a lesões no ombro (Moller, 2017). Essas descobertas sugerem que grandes aumentos semanais na carga do handebol são o principal fator de risco para lesões no ombro, além disso o tênis precisa de métodos validados de monitoramento da carga de trabalho para examinar o comportamento das cargas durante o treinamento e as partidas para identificar uma possível conexão com lesões. Há importância no gerenciamento ideal da carga de treinamento, fazendo-se necessário quantificar a carga de treino e adaptá-la a cada atleta. A frequência das partidas esportivas e a intensidade do treinamento têm uma influência importante nos problemas do ombro, ampliando as lesões por esforço causadas por movimentos inadequados e falta de tempo de recuperação (Tooth, 2020; Moller, 2017; Kekelekis, 2020).

Ao analisar os fatores de riscos potenciais para causar lesão de membro superiores em tenistas pode-se observar que os principais são: tênis prolongado, discinesia escapular, fadiga muscular, propriedades escapulotorácicas, cinemática do ombro, nível de habilidade e técnica. Os tenistas com discinese escapular tendem a apresentar redução do escapo subacromial, a discinesia escapular pode reduzir o espaço subacromial, embora as evidências para isso sejam confusas, também pode reduzir a força do manguito rotador, essa pode prejudicar o controle motor, resultando na translação superior e posterior da cabeça do úmero gerando um desgaste mecânico das estruturas do espaço subacromial (Kekelekis, 2020; Hickey, 2017).

Estudos corroboram para o fato da discinese escapular possuir diversos efeitos que podem alterar o funcionamento ideal do ombro. Incluindo o aumento do Ângulo da glenoumeral em abdução horizontal, consecutivamente aumenta a tensão capsular anterior e o cisalhamento, levando a aumentos na compressão labral posterior, diminuindo a força do manguito rotador, diminuindo o espaço subacromial e diminuindo (Kibler, 2019). Uma boa coordenação dos grupos musculares acontece de forma dinâmica e é adaptada de acordo com a tarefa que será realizada e conforme os requisitos de estabilidade. O deltoide gera uma força desestabilizadora durante os graus iniciais de elevação do ombro, o manguito rotador, grande dorsal e redondo maior são ativados com a função de equilibrar a atividade muscular. Na amplitude final do movimento, a demanda por estabilidade muscular aumenta novamente sendo fornecida pelo manguito rotador (Hawkes, 2019).

Quando analisamos a prevalência de discinese em uma população de atletas juvenis com nível esportivo amador, obtemos resultado relativamente alto, no entanto a relação dor e alteração funcional do membro superior é deficitária e os estudos não corroboram para uma conclusão precisa. Alguns estudiosos ainda afirmam que a discinese escapular não deve ser considerado um sinal patológico, mas sim um mecanismo compensatório para os indivíduos que utilizam intensamente o membro superior, isso devido a adaptações das estruturas (Oliveira, 2018).

A reabilitação escapular deve envolver ativação seletiva dos músculos mais fracos e na ativação mínima dos músculos hiperativados, assim a escápula pode recuperar sua função como uma base estável de suporte para o úmero durante um arremesso por cima. Sendo recomendados exercícios de cadeia aberta em plano único abaixo de $90^{\circ}$ de elevação do ombro, a fita rígida e Kinesio a curto prazo pode melhorar o comprimento menor do peitoral e a discinesia escapular em atletas de arremesso assintomáticos, a incorporação de exercícios em cadeia cinética tem sido sugerida para influenciar os níveis de atividade da musculatura escapular. Realizar uma elevação bilateral com rotação externa usando uma mão aberta em vez de fechada é mais benéfico quando uma maior atividade da musculatura escapular é desejada na reabilitação (Borms, 2020; Ozer, 2019).

\section{Conclusão}

A discinese escapular comumente está presente em esportes específicos para membros superiores, sendo que idade, volume de treinamentos e cargas podem aumentar as chances de lesões no membro superior quando comparados a atletas sem alterações na movimentação escapular. Ressaltando que a alteração na movimentação escapular pode não ser necessariamente 
algo patológico, mas compensatório devido a demanda exigida da estrutura da cintura escapular, fazendo-se necessários mais estudos sobre a temática.

Apesar da importante variabilidade da qualidade dos estudos, as modalidades encontradas abordaram atletas juvenis de voleibol, natação, handebol, basquete, judô e jogadores de tênis de diferentes idades, gêneros e níveis de habilidade. Dessa forma, destaca-se a importância deste estudo no contexto acadêmico e profissional, entendendo que através dos resultados obtidos ambos os campos terão essa lacuna sanada, enriquecendo a análise e pensamento clínico, consequentemente ocorrerá uma potencializando na prática baseada em evidências. Através dos achados desse estudo observa-se relevante a recomendação de novos estudos, com maiores amostragens e diferentes critérios para exclusão e inclusão, afim de proporcionar uma maior evidência científica sobre a temática.

\section{Referências}

Andersson, S. H., Bahr, R., Clarsen, B., \& Myklebust, G. (2016). Preventing overuse shoulder injuries among throwing athletes: A cluster-randomised controlled trial in 660 elite handball players. British Journal of Sports Medicine, 51(14), 1073-1080. https://doi.org/10.1136/bjsports-2016-096226

Borms, D., Maenhout, A., \& Cools, A. M. (2020). Incorporation of the kinetic chain into shoulder-elevation exercises: Does it affect scapular muscle activity? Journal of Athletic Training, 55(4), 343-349. https://doi.org/10.4085/1062-6050-136-19

Ercole, F. F., Melo, L. S. d., \& Alcoforado, C. L. G. C. (2014). Integrative review versus systematic review. Reme: Revista Mineira de Enfermagem, 18(1). https://doi.org/10.5935/1415-2762.20140001

Giuseppe, L. U., Laura, R. A., Berton, A., Candela, V., Massaroni, C., Carnevale, A., Stelitano, G., Schena, E., Nazarian, A., DeAngelis, J., \& Denaro, V. (2020). Scapular dyskinesis: From basic science to ultimate treatment. International Journal of Environmental Research and Public Health, 17(8), 2974. https://doi.org/10.3390/ijerph17082974

Hawkes, D. H., Khaiyat, O. A., Howard, A. J., Kemp, G. J., \& Frostick, S. P. (2019). Patterns of muscle coordination during dynamic glenohumeral joint elevation: An EMG study. Plos One, 14(2), Artigo e0211800. https://doi.org/10.1371/journal.pone.0211800

Hejrati, B., Chesebrough, S., Bo Foreman, K., Abbott, J. J., \& Merryweather, A. S. (2016). Comprehensive quantitative investigation of arm swing during walking at various speed and surface slope conditions. Human Movement Science, 49, 104-115. https://doi.org/10.1016/j.humov.2016.06.001

Hickey, D., Solvig, V., Cavalheri, V., Harrold, M., \& Mckenna, L. (2017). Scapular dyskinesis increases the risk of future shoulder pain by 43\% in asymptomatic athletes: A systematic review and meta-analysis. British Journal of Sports Medicine, 52(2), 102-110. https://doi.org/10.1136/bjsports-2017097559

Huang, T.-S., Du, W.-Y., Wang, T.-G., Tsai, Y.-S., Yang, J.-L., Huang, C.-Y., \& Lin, J.-J. (2018). Progressive conscious control of scapular orientation with video feedback has improvement in muscle balance ratio in patients with scapular dyskinesis: A randomized controlled trial. Journal of Shoulder and Elbow Surgery, 27(8), 1407-1414. https://doi.org/10.1016/j.jse.2018.04.006

Kekelekis, A., Nikolaidis, P. T., Moore, I. S., Rosemann, T., \& Knechtle, B. (2020). Risk factors for upper limb injury in tennis players: A systematic review. International Journal of Environmental Research and Public Health, 17(8), 2744. https://doi.org/10.3390/ijerph17082744

Kibler, W. B., \& Sciascia, A. (2019). Evaluation and management of scapular dyskinesis in overhead athletes. Current Reviews in Musculoskeletal Medicine, 12(4), 515-526. https://doi.org/10.1007/s12178-019-09591-1

McQuade, K. J., Borstad, J., \& de Oliveira, A. S. (2016). Critical and theoretical perspective on scapular stabilization: What does it really mean, and are we on the right track? Physical Therapy, 96(8), 1162-1169. https://doi.org/10.2522/ptj.20140230

Meron, A., \& Saint-Phard, D. (2017). Track and field throwing sports. Current Sports Medicine Reports, 16(6), 391-396. https://doi.org/10.1249/jsr.0000000000000416

Møller, M., Nielsen, R. O., Attermann, J., Wedderkopp, N., Lind, M., Sørensen, H., \& Myklebust, G. (2017a). Handball load and shoulder injury rate: A 31week cohort study of 679 elite youth handball players. British Journal of Sports Medicine, 51(4), 231-237. https://doi.org/10.1136/bjsports-2016-096927

Møller, M., Nielsen, R. O., Attermann, J., Wedderkopp, N., Lind, M., Sørensen, H., \& Myklebust, G. (2017b). Handball load and shoulder injury rate: A 31week cohort study of 679 elite youth handball players. British Journal of Sports Medicine, 51(4), 231-237. https://doi.org/10.1136/bjsports-2016-096927

Mulvad, B., Nielsen, R. O., Lind, M., \& Ramskov, D. (2018). Diagnoses and time to recovery among injured recreational runners in the RUN CLEVER trial. Plos One, 13(10), Artigo e0204742. https://doi.org/10.1371/journal.pone.0204742

Nodehi Moghadam, A., Rahnama, L., Noorizadeh Dehkordi, S., \& Abdollahi, S. (2020). Exercise therapy may affect scapular position and motion in individuals with scapular dyskinesis: A systematic review of clinical trials. Journal of Shoulder and Elbow Surgery, 29(1), Artigo e29-e36. https://doi.org/10.1016/j.jse.2019.05.037

Nowotny, J., Kasten, P., Kopkow, C., Biewener, A., \& Mauch, F. (2018). Evaluation of a new exercise program in the treatment of scapular dyskinesis. International Journal of Sports Medicine, 39(10), 782-790. https://doi.org/10.1055/a-0608-4584 
Research, Society and Development, v. 10, n. 16, e542101623987, 2021

(CC BY 4.0) | ISSN 2525-3409 | DOI: http://dx.doi.org/10.33448/rsd-v10i16.23987

Oliveira, V. M. A. d., Silva, H. A. d., Pitangui, A. C. R., Passos, M. H. P. d., \& Araújo, R. C. d. (2018). Scapular dyskinesis was not associated with pain and function in male adolescent athletes. Revista Dor, 19(1). https://doi.org/10.5935/1806-0013.20180008

Ozer, S. T., Karabay, D., \& Yesilyaprak, S. S. (2018). Taping to improve scapular dyskinesis, scapular upward rotation, and pectoralis minor length in overhead athletes. Journal of Athletic Training, 53(11), 1063-1070. https://doi.org/10.4085/1062-6050-342-17

Romkes, J., \& Bracht-Schweizer, K. (2017). The effects of walking speed on upper body kinematics during gait in healthy subjects. Gait \& Posture, 54, 304310. https://doi.org/10.1016/j.gaitpost.2017.03.025

Tooth, C., Gofflot, A., Schwartz, C., Croisier, J.-L., Beaudart, C., Bruyère, O., \& Forthomme, B. (2020). Risk factors of overuse shoulder injuries in overhead athletes: A systematic review. Sports Health: A Multidisciplinary Approach, 12(5), 478-487. https://doi.org/10.1177/1941738120931764 